\title{
Implementation of Assertive Alcohol Screening in a Gastroenter- ology Outpatients Department of an Acute Hospital
}

\author{
${ }^{1}$ University of Surrey, United Kingdom \\ ${ }^{2}$ West Middlesex University Hospital, United Kingdom \\ ${ }^{3}$ IHEAR Partnership, United Kingdom
}

Maya Knox-Macaulay ${ }^{*}$, Nina Stafford ${ }^{2}$, Carole Collins ${ }^{2}$ and Christos Kouimtsidis ${ }^{3}$

*Corresponding author: Maya Knox-Macaulay, B.Sc. Student, University of Surrey, United Kingdom, Tel: +447730 876558,E-mail:mk00269@surrey.ac.uk

\begin{abstract}
There is a gap in the literature when considering the impact of screening using AUDIT-C, the shortened version of the AUDIT, to improve records of alcohol intake in a secondary care environment. Many previous studies have looked into the efficacy and financial cost of AUDIT screening in primary, secondary and emergency care settings. However, in a time and cost limited outpatient's department, implementation of the full AUDIT is not viable. The AUDIT-C is a tool which hasn't been fully utilised by NHS hospitals as a method of detecting and limiting harmful alcohol use in the outpatient population. The following article is a summary of a study to investigate the feasibility of implementing focused and consistent alcohol screening within a gastroenterology outpatients department, an appropriate secondary care environment. It specifically considers the barriers and facilitators in regards to patients and staff members.

The primary purpose of the research was to identify the quantitative level of compliance in patients who were delivered the AUDIT-C. Appropriate training is required for effective implementation of assertive screening to be carried out. Further research is required to cement the findings, potentially by broadening implementation to other outpatients' departments at West Middlesex University Hospital (WMUH).
\end{abstract}

\section{Keywords}

Alcohol screening, Acute hospital, Gastroenterology outpatient

\section{Introduction}

Alcohol misuse is prolific; the yearly cost of alcohol related conditions to the NHS sits at approximately $£ 3.5$ billion [1]. Intrinsically though, the cost is primarily to patient health. The extent to which alcohol misuse can impact on the body varies from person to person. Alcohol could be the sole primary cause of a medical condition or could contribute to complex multifactorial disease. Aside from this, alcohol misuse can be an independent issue, which if undetected and handled appropriately at the time could lead to further health complications, generating more pressure on the NHS. It is known that alcohol can have a harmful effect on most of the organs and organ systems of the human body and in particular the association between liver disease and alcohol is well recognised [2].

The need for alcohol screening has long been recognised and recommended. A 2001 report from the Royal College of Physicians suggested that screening strategies should be implemented through lifestyle questionnaires as a method to detect harmful or hazardous drinking at an earlier stage. Another recommendation was to provide brief interventions (BIs) to patients drinking at hazardous levels and referral to appropriate support services, if required [3]. In support of this, the All Party Parliamentary Hepatology Group (APPHG) Inquiry into Improving Outcomes in Liver Disease, identified through Alcohol Concern that one opportunity to approach the issue of liver disease prevention in the NHS could be through brief intervention, which they imply is shown to be useful in decreasing the levels of alcohol consumption in patients seen in emergency services and on general hospital wards [4].

These are both further supported by the National Institute for Health and Care Excellence (NICE) guidelines 
for 'Alcohol-use disorders: diagnosis and management'. In the quality statement for the use of brief interventions and screening in practice, they recommend that 'Health and social care staff opportunistically carry out screening and brief interventions for hazardous and harmful drinking as an integral part of practice' [5]. This echoes the sentiment of the previous report, in that effective screening, intervention, assessment and treatment is the process in which alcohol misuse can be best managed. Unfortunately, even with these guidelines in place, screening is still not implemented in a consistent way.

The 1998 General Household Survey summated that just over one in seven women (15\%) and approximately one in four men $(27 \%)$ drink over the recommended weekly guidelines for alcohol consumption. This issue is not restricted to adults; the age category with the heaviest drinkers is $16-24$ year olds, with over a third (36\%) of young men drinking more than 21 units a week and a quarter of young women drinking more than 14 units a week [6]. This report also indicated that one of the major barriers in developing strategies to combat harmful drinking is that the consumption of alcohol is indicated to have some health benefits. The recent guidelines developed by the UK Chief Medical Officers suggest concrete safe levels of drinking; these may be misinterpreted in signifying that there is not an increased risk to the person, if their alcohol intake is below this-a wholly disputed assumption [3].

The 2010 review of alcohol misuse screening identified organisational, staff and patient barriers/facilitators which can occur in primary, secondary and emergency care settings. The article indicated that staff attitudes to health promotion were found to be generally positive, but also noted the barriers identified through the reviewed studies [7]. Lock, et al. determined that receptionists had a less positive attitude to handing out questionnaires in comparison to clinicians, when not reimbursed for their time [8]. In a study by McManus, et al. barriers to implementation occurred when there was conflict about what a clinician's role should involve. Also, it was seen that training nursing staff in interventions was found to be more acceptable when it was delivered by another nurse [9]. Culturally, there are varying outlooks on alcohol; a survey by Heim, et al. indicated that alcohol misuse was often under-reported in the Indian community [10].

The direct burden that alcohol misuse has on the NHS emergency services is clear, but the effect on secondary services including inpatient and outpatient care is not so obvious. The purpose of our study was to investigate the potential barriers to and facilitators of implementing alcohol screening for all patients attending an outpatient gastroenterology department of an acute hospital trust; the study was informed by the NICE guidelines for screening and intervention, specifically quality statements 2 and 3 and the commissioning guideline $3.1[5,11]$. If implementation was effective, then use of the screening form would lead to an opportunity for Bl, by the clinicians. Alongside this, increased access to the Alcohol Liaison services could therefore reduce the number of re-admittances to the outpatients department for ongoing health issues due to alcohol abuse. The main objectives were to:

1. Revise the alcohol screening form (Figure 1), which includes the AUDIT-C (Alcohol Use Disorder Identification Test-Consumption)-the shortened version of the AUDIT (Alcohol Use Disorder Identification Test) questionnaire.

2. Collect quantitative data on compliance in screening form uptake.

3. Devise a follow up questionnaire for both patients and nursing staff.

4. Collect qualitative data on the follow up questionnaire to assess barriers in patients and nursing staff.

5. Handover responsibility for screening patients to nursing staff.

\section{Methods}

\section{Procedures}

The methodology was discussed and agreed between the gastroenterology team and the alcohol liaison team. The point at which the screening form should be delivered was discussed to standardise practice. Three main options were considered: (i) to send the form with the appointment letter; (ii) to give to patient on their arrival in the clinic waiting area; or (iii) to hand over to patient whilst the clinic nurse was performing, routine observations (i.e. height, weight and blood pressure). The first option was ruled out because it was considered that this could cause the patient to feel stigmatised and even fail to attend. The waiting room was a plausible option because it would not be disruptive to the clinical staff however confidentiality could be compromised and ensuring delivery to every patient could be challenging. On the other hand, although there may be time pressures, the observations room has the clear advantage of confidentiality and reliable delivery. It was initially agreed that it should be the responsibility of an interviewer (M $\mathrm{K}-\mathrm{M})$ to distribute the screening form to the gastroenterology patients, and if successful this responsibility could be passed to the nursing staff. Later a semi-structured follow up survey was designed to explore any barriers which could arise. The follow up survey would be carried out in a separate room, if available.

\section{Setting}

Two general gastroenterology clinics occur every week within the Gastroenterology outpatients department of a teaching hospital in London. In the guidelines, it specifies that in an outpatient clinic setting, the patients who are most appropriate for screening are those with cardiac, gastrointestinal and neurological disorders [12]. This guided the decision to use the gastroenterology outpatients department as the first location. 


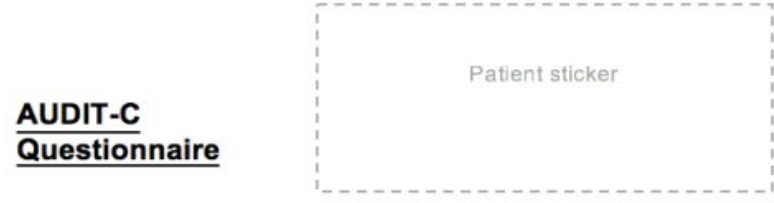

Please answer the following questions about your alcohol consumption, to check if your drinking is within healthy limits: (see back of leaflet for unit guidelines)

\begin{tabular}{|c|c|c|c|c|c|c|}
\hline \multirow{2}{*}{ Questions } & \multicolumn{5}{|c|}{ Scoring system } & \multirow{2}{*}{$\begin{array}{l}\text { Your } \\
\text { score }\end{array}$} \\
\hline & 0 & 1 & 2 & 3 & 4 & \\
\hline $\begin{array}{l}\text { How often do } \\
\text { you have a } \\
\text { drink } \\
\text { containing } \\
\text { alcohol? }\end{array}$ & Never & $\begin{array}{l}\text { Monthly } \\
\text { or less }\end{array}$ & $\begin{array}{c}2-4 \\
\text { times } \\
\text { per } \\
\text { month }\end{array}$ & $\begin{array}{c}2-3 \\
\text { times } \\
\text { per } \\
\text { week }\end{array}$ & $\begin{array}{c}4+ \\
\text { times } \\
\text { per } \\
\text { week }\end{array}$ & \\
\hline $\begin{array}{l}\text { How many } \\
\text { units of alcohol } \\
\text { do you drink on } \\
\text { a typical day } \\
\text { when you are } \\
\text { drinking? }\end{array}$ & $1-2$ & $3-4$ & $5-6$ & $7-9$ & $10+$ & \\
\hline \multirow[t]{2}{*}{$\begin{array}{l}\text { How often have } \\
\text { you had } 6 \text { or } \\
\text { more units if } \\
\text { female, or } 8 \text { or } \\
\text { more if male, } \\
\text { on a single } \\
\text { occasion in the } \\
\text { last year? }\end{array}$} & Never & $\begin{array}{l}\text { Less } \\
\text { than } \\
\text { monthly }\end{array}$ & Monthly & Weekly & $\begin{array}{l}\text { Daily } \\
\text { or } \\
\text { almost } \\
\text { daily }\end{array}$ & \\
\hline & & & & \multicolumn{2}{|c|}{ Total score } & \\
\hline
\end{tabular}

I give my consent for the Gastroenterology team to refer me to the Alcohol Liaison Service, if required:
Score:

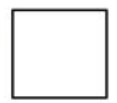

If scoring $\mathbf{4}$ or less: You are drinking within guidelines and are less likely to develop alcohol related problems.

If scoring $\mathbf{5}$ or above: You are potentially drinking too much and could be putting your health at risk. Try to reduce your alcohol consumption to safer levels using the safe consumption strategies below, and consider whether or not you may require professional advice/support with this.

If scoring 12: Please phone the Alcohol Liaison Officers within the hospital on 07826910162 , who will see you on the same day, or if you prefer we can make an external appointment at the iHEAR Partnership on 02085381150

\section{Safe consumption strategies}

- Have 2-3 days per week without drinking alcohol. This will provide your body with some recuperation.

- When drinking do not exceed levels below: Women: 2-3 units daily; Men: 3-4 units daily.

- If you attend a special occasion i.e. birthday, or other event, try not to consume more than $6-8$ units, as this could lead to future complications with your emotional and or physical wellbeing.

Ticking the box on the opposite page enables you to give your consent for the Gastroenterology team to refer you to the Alcohol Liaison services, if required.

iHEAR Partnership,

Pharmacia House, 1 Prince Regent Road, Hounst
TW3 1 NE

Tel No. 02085381150

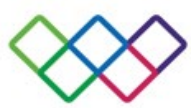

\section{Healthy Drinking}

In line with Public Health England guidelines, West Middlesex University Hospital is committed to promoting healthy attitudes towards alcohol and safe consumption amongst all our patients and their relatives.

Please assist us by answering the following short questionnaire and then hand the completed form to the doctor you see today.

If you have any queries, don't hesitate to ask a member of staff for assistance.

\section{Instruments}

\section{Alcohol screening form}

The screening form included the AUDIT-C, explanation and advice about the score and a questionnaire on factors that might be a barrier to screening implementation. The AUDIT-C is a shortened version of the full AUDIT screening questionnaire, made up of three questions. A score of 4 and below suggests that a person is drinking within safe levels. A score of $5+$ indicates that a person is drinking at increased risk levels, and should try and reduce their alcohol consumption to a safer level. A score of $8+$ indicates higher risk of hazardous and harmful drinking. This screening form follows the Public Health England guidelines, a standard endorsed by the NHS [13]. Within their commissioning guidelines (3.1), NICE mentions the importance in using appropriate screening tools, specifying in particular, the AUDIT (AIcohol Use Disorder Identification Test) where viable and AUDIT-C (Alcohol Use Disorder Identification Test - Con- 
sumption) for time-pressured interactions [11]. It conforms to the International Classification of diseases 10 (ICD-10) and has been approved for international use, which indicates that it follows a format which can be applied in a variety of diverse healthcare systems [12].

There are no written guidelines to inform about appropriate use of the AUDIT-C in comparison to the full AUDIT, so the AUDIT-C guidelines have been employed for use as a directive within this pilot study.

Also included in the screening form was an explanation of the calculated score, with higher scores directing the patient to two sources of assistance: safe consumption strategies and contact details for the Alcohol Liaison Service (ALS). An optional consent box was added at the end of the form, to enable the gastroenterology team to refer any patients at risk due to the quantity of alcohol they were consuming.

\section{Patient survey}

A questionnaire was developed collecting information about some of the predicted barriers into the implementation of the alcohol screening such as ethnicity, age, sex, language and religious beliefs, as well as questions about patient's opinion about the screening. The most recent 2011 census details for the local borough of Hounslow were investigated, specifically for language and ethnicity. After 'White British' (37.9\%) it indicated that the next most prominent ethnicities were 'Indian' (19.0\%), 'Other White' (11.5\%) and 'Other Asian' (8.2\%) [14].

\section{Staff survey}

To examine barriers associated with staff attitudes in depth, a semi-structured qualitative questionnaire was delivered to 2 regular members and 1 bank member of nursing staff.

\section{Procedure}

The screening form was handed to every appropriate Gastroenterology patient over eleven outpatient clinic sessions during a three-month period (September-November 2015). One researcher (M K-M) assisted the patients in completing it whilst they were having their height, weight and blood pressure measured by the clinic nurse.

The census also presented data which showed that in 17.1\% of households within Hounslow no one speaks English as their main language [14]. Therefore, we expected to encounter culture and language barriers present in some of the patient/staff interactions. To approach these potential barriers, we did our best to convey the AUDIT-C in the clearest manner possible. When there were language barriers present, we used family or nursing staff to translate the questions. Sometimes patients would use translators, or the questions could be described differently to aide their understanding. To make the study culturally appropriate, we considered the questions sensitively, especially in relation to the age and ethnicity of the patients.

After three months, the responsibility for handing out the screening form was passed to the clinic nurses. This was overseen and support for staff was provided for a further six clinics.

\section{Results}

\section{Implementation of screening}

Across the eleven clinics attended the researcher ( $M$ $\mathrm{M}-\mathrm{K}$ ) was present at 258 of the nurse/patient clinical interactions, from which 205 were eligible to take part in this project. Fifty three patients were from other clinics such as Neurology, Haematology and Colorectal (Figure 2).

From the remaining of 205 gastroenterology patients, 200 agreed to complete the screening form, $97.6 \%$ of the total gastroenterology patients attending the clinics.

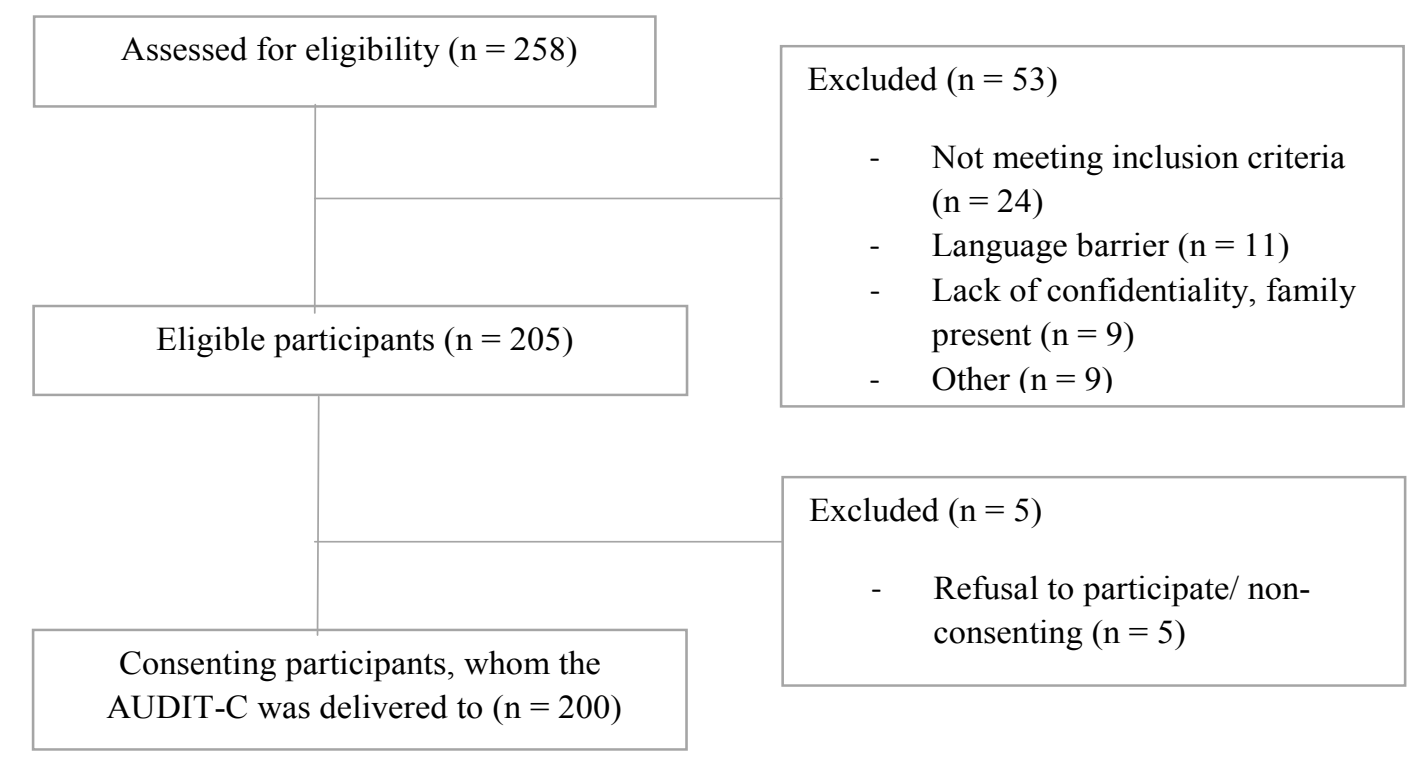

Figure 2: CONSORT diagram detailing the eligibility of patients for delivery of the AUDIT-C within the gastroenterology outpatients department, WMUH. 
One hundred and forty one patients $(69 \%)$ have completed the AUDIT-C, with over half $(72 / 141)$ having a score of 0 , indicating abstinence or very minimal alcohol consumption. Some of these patients showed resistance in filling out the screening form, they felt it was inappropriate because they were not consuming alcohol.

Pre (August 2015) and post (December 2015) implementation of alcohol screening, comparison of the information recorded by the Gastroenterology department of alcohol intake and the number of Bls offered has increased from $54 \%$ to $69 \%$ and from $2.6 \%$ to $10.1 \%$ respectively. The number of referrals to the Alcohol Liaison Service has not changed.

\section{Patient survey}

Eighteen patients have completed the patient questionnaire assessing barriers for implementation of alcohol screening, with equal male and female participation. All but one participant were between the ages of 25-64, and one participant older than 75 years. 'White' was the most prevalent ethnicity (11 patients), followed by mixed ethnicities (5 patients), one Black/African/ Caribbean and one Asian patient. All patients reported receiving the alcohol screening form and completing it. All patients reported that they felt comfortable with answering the questions on the alcohol screening form. Seventeen participants (94.4\%) agreed that patients should be asked about their alcohol consumption when they attend the gastroenterology clinic. All patients believed that there is a relationship between alcohol and liver problems. Fourteen patients stated they would prefer to complete the alcohol screening at the clinic, with only 3 patients preferring the option of completing it at home, and one participant having no preference.

\section{Staff survey}

The questionnaire delivered to the staff members indicated that they all agreed that they would feel comfortable handing out the alcohol screening form to patients. Nevertheless, they reported that the presence of the interviewer would enable them to remember to do it. They all agreed that alcohol has implications on the health of a patient with a gastroenterological disease and that the screening form should become an integrated aspect of patient care in the gastroenterology unit. One nurse stated 'Most patients are unaware of damage caused by alcohol-if they are educated, it will benefit both (the) patients and (the) NHS'.

\section{Discussion}

The results of this study indicate that there was excellent compliance $(97.5 \%)$ by patients in filling out the alcohol screening form (AUDIT-C with advice on scoring and action required). This closely follows the results of the patient survey, where all but one patient felt comfortable answering the screening form. Only one patient thought that others should not be asked about their alcohol intake when they visited the gastroenterology outpatients unit. This contrasts to the original concern expressed by clinic staff during the designing phase of this project that there might be patient reluctance to participate.

All patients correctly indicated that there was in fact a relationship between liver conditions and alcohol, implying that patients are aware that alcohol can cause harm to the human body. However, the survey was delivered to a very small proportion of the patients $(8.8 \%)$, over one clinic session, due to restrictions on the availability of the separate room. To fully explore the barriers in patient compliance, further research needs to be carried out, to determine if these inferences are accurate and truly represent the views of the patients passing through the gastroenterology outpatient department.

A specifically developed training programme might help to overcome staff resistance to implement alcohol screening. Consideration should be given to alert staff and patients to the clear link between the effect of alcohol and the specialty of the department.

\section{Acknowledgement}

Authors would like to thank the staff and patients attending the gastroenterology outpatient clinics at WMUH.

\section{Ethical statement}

No ethics approval was required for this study. All authors confirm no conflict of interest.

\section{References}

1. (2014) Alcohol treatment in England 2013-14. Public Health England: HM Government.

2. Maher JJ (1997) Exploring Alcohol's Effects on Liver Function: Types Of Alcohol-Induced Liver Damage. Alcohol Health \& Research World 21: 5-12.

3. Royal College of Physicians (2001) A report of a working party of the royal college of physician's recommendations for a coherent alcohol strategy for hospitals. ALCOHOL - can the NHS afford it? Sarum ColourView Group, Salisbury, Wiltshire: Publications Unit of the Royal College of Physicians.

4. Liver Disease: Today's complacency, tomorrow's catastrophe.

5. NICE (2011)Alcohol-use disorders: Diagnosis and management.

6. Bridgwood A, Lilly R, Thomas M, Bacon J, Sykes W, et al. (1998) Living in Britain results from the 1998 general household survey, UK.

7. Johnson M, Jackson R, Guillaume L, Meier P, Goyder E (2010) Barriers and facilitators to implementing screening and brief intervention for alcohol misuse: A systematic review of qualitative evidence. J Public Health (Oxf) 33: 417.

8. Lock CA, Kaner EF, Heather N, Gilvarry E, McAvoy BR (2000) Changes in receptionists attitudes towards involvement in a general practice-based trial of screening and brief alcohol intervention. Br J Gen Pract 50: 111-115.

9. McManus S, Hipkins J, Haddad P, Guthrie E, Creed F (2003) Implementing an effective intervention for problem drinkers on medical wards. Gen Hosp Psychiatry 25: 332-337.

10. Heim D, Hunter SC, Ross AJ, Bakshi N, Davies JB, et al. (2004) Alcohol consumption, perceptions of community 
responses and attitudes to service provision: results from a survey of indian, chinese and pakistani young people in Greater Glasgow, Scotland, UK. Alcohol and Alcoholism 39: 220-226.

11. https://www.nice.org.uk/guidance/QS11/resources/servicesfor-the-identification-and-treatment-of-hazardous-drinkingharmful-drinking-and-alcohol-dependence-in-childrenyoung-people-and-adults-commissioning-guide-304112845/ chapter/31-Opportunistic-screening-and-brief-interventionsfor-adults-who-are-hazardous-and-harmful-drinkers
12. Babor TF, Higgins-Biddle JC, Saunders JB, Monteiro MG (2001) The alcohol use disorders identification test guidelines for use in primary care. (2nd edn), World Health Organization, Switzerland.

13. AUDIT - C - latest resources - resources - PHE alcohol learning resources.

14. Hounslow (2013) Ethnicity, national identity and language census data. 